\section{JTI}

JOURNAL OF

TRAUMA AND INJURY

\title{
Pulmonary Thromboembolism during Acetabular Fracture Operation
}

\author{
Il Seo, M.D. ${ }^{1,2}$, Chang-Wug Oh, M.D., Ph.D. ${ }^{1,2}$, Joon-Woo Kim, M.D., Ph.D. ${ }^{1,2}$, \\ Kyeong-Hyun Park, M.D. ${ }^{1,2}$ \\ ${ }^{1}$ Regional Trauma Center, Kyungpook National University Hospital, Daegu, Korea \\ ${ }^{2}$ Department of Orthopaedic Surgery, School of Medicine, Kyungpook National University, \\ Daegu, Korea
}

Received: May 31, 2018

Revised: July 2, 2018

Accepted: July 4, 2018

\section{Correspondence to}

Chang-Wug Oh, M.D., Ph.D.

Department of Orthopedic Surgery, Kyungpook National University Hospital, School of Medicine, Kyungpook National University, 130 Dongdeok-ro, Jung-gu,

Daegu 41944, Korea

Tel: $+82-53-420-5630$

Fax: +82-53-422-6605

E-mail:cwoh@knu.ac.kr
Acetabular and Pelvic ring fractures are major high-energy trauma injuries and are often combined with other injuries. In particular, cause of long duration of immobilization and combined injuries, venous thromboembolism is a common complication in trauma patients with pelvic or acetabular fractures. We report a case of a fatal pulmonary thromboembolism during the acetabulum fracture operation in a 62-year-old male patient.

Keywords: Pulmonary thromboembolism; Acetabulum; Wounds and injuries

\section{INTRODUCTION}

Acetabular fractures and pelvic ring injury are commonly caused by high energy trauma and are often almost combined with other injuries. Consequently, prolonged periods of immobilization are needed. This in turn, risks the can be risk factors for the development of thromboembolism [1,2]. Deep vein thrombosis (DVT) after fracture of the pelvis has been reported to occur up to $61 \%$ of the time [3] and the pulmonary thromboembolism (PTE) after pelvic trauma up to $10 \%$ of the time. Furthermore, a fatal event was reported in half of this population [3,4].

Symptoms of pulmonary embolism which include chest pain, irregular heartbeats, dizziness, rapid breathing, cough, etc. are unspecific. Often times, the first clinical manifestation of venous thromboembolism (VTE) is PTE-related sudden death. Due to delayed diagnosis, sudden onset of symptoms, their unpredictable nature and rapid progression, patients are subject to expire frequently when this complication occurs.

The authors experienced a case of an acute PTE during an acetabular fracture operation in a previously healthy man whose life was saved by an immediate diagnosis of (http://creativecommons.org/licenses/by-nc/4.0/) which permits unrestricted noncommercial use, distribution, and reproduction in any medium, provided the original work is properly cited. 
PTE and surgical thromboembolectomy by thoracic surgeon. So, we report with further literature review.

\section{CASE REPORT}

A previously healthy 62 -year-old man sustained a fall from a height of about 3 meters while picking persimmon and 3 days after the injury he was transferred to our institution via a local medical center. Initial vital signs were relatively stable. Clinical and radiological assessment revealed a right acetabular anterior column with anterior wall fracture, right iliac wing fracture and no other combined injury (Fig. 1).

The patient had bed rest with skeletal traction for the acetabulum fracture and a chest X-ray was checked daily. To prevent possible VTE, mechanical prophylaxis with anti-embolic stocking and an intermittent pneumatic compression pump was performed. After 10 days from the time of injury, the patient's condition had improved and the surgery was held. The plan was to operate open reduction and fixation for acetabulum. About 45 minutes after starting the operation of the acetabulum fracture under the general anesthesia, the patient's blood pressure (BP) rapidly became hypotensive (systolic BP/diastolic BP: 30/20). Peripheral pulsation became weak and eventually uncheckable. During the assessment of situation by the anesthesiologist, cardiac arrest occurred and all operative procedures were stopped. Cardiopulmonary resuscitation

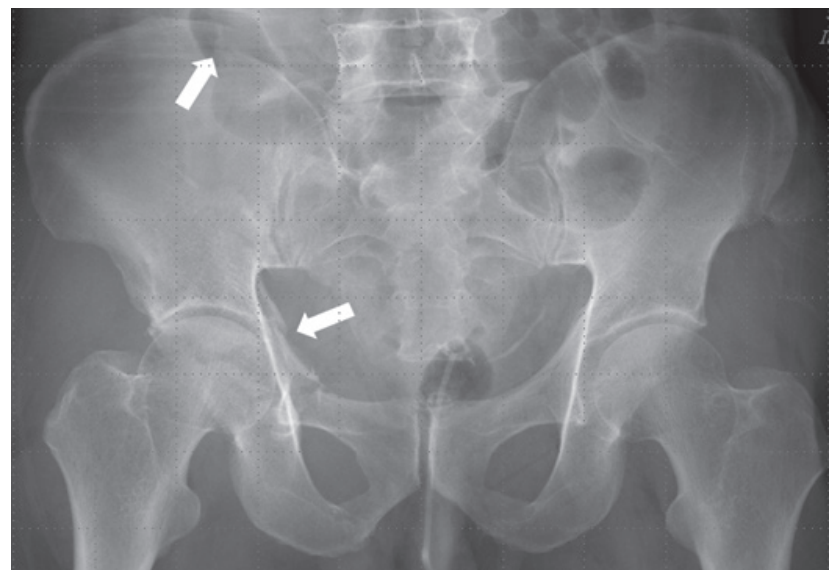

Fig. 1. Right acetabular anterior column with anterior wall fracture, right iliac wing fracture. Arrows show acetabulum fracture line. was conducted and heart rhythm was restored to sinus rhythm after cardiac massage for 3 minutes, but oxygen saturation and blood pressure were not fully regained. A transesophageal echocardiography was carried out immediately in the operating room and showed no thrombi in his atrium and ventricle (Fig. 2). Left ventricular function was relatively maintained without regional wall motion abnormality and no thrombi in the cardiac chamber but was slightly interfered with the dilated right ventricle.

Chest computed tomography (CT) scan was done to find cause of arrest. Massive thromboembolism on both pulmonary arteries were found (Fig. 3). Acute PTE was diagnosed and immediate pulmonary artery embolectomy was done by a thoracic surgeon with sternotomy.

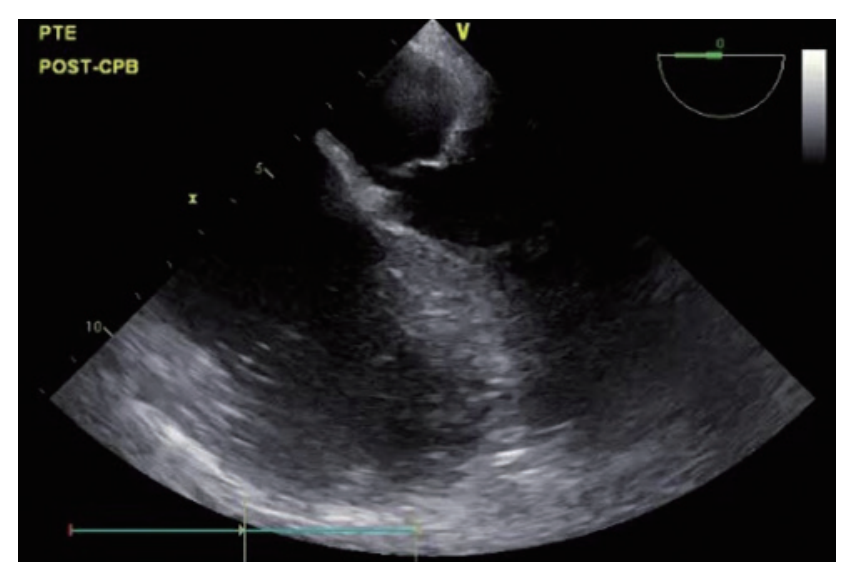

Fig. 2. Intra-operative cardiac echo graphs. Shows no thrombi in the cardiac chamber.

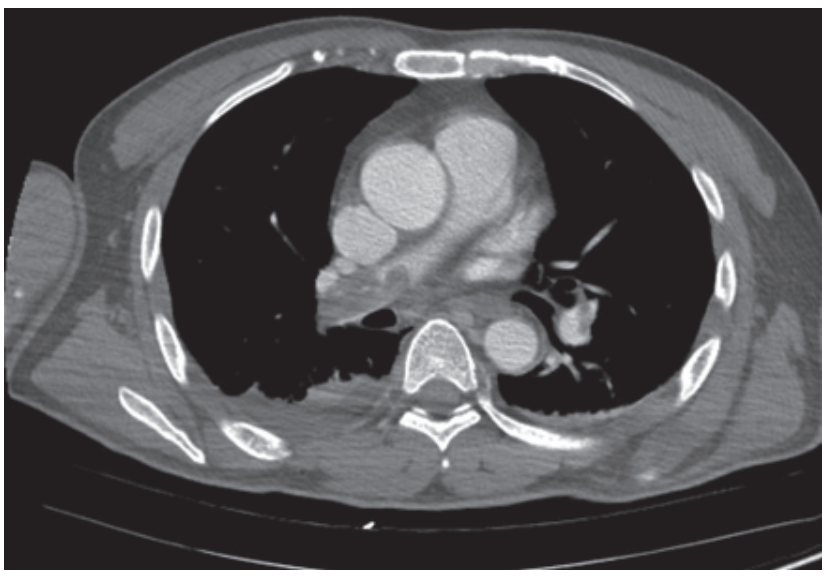

Fig. 3. CT shows massive thromboemboli in both pulmonary artery. CT: computed tomography. 
Surgery was performed through median sternotomy with aortic \& right atrial cannulation and normothermic cardiopulmonary bypass (CPB). Surgery was performed bilateral transverse incision. This incision can be extended transversely onto the main pulmonary artery to improve each-side exploration. Clot is extracted with a combination of gallbladder stone forceps and suction. Decreasing cardiopulmonary bypass flows aids clot visualization.

Four pieces of large emboli were evacuated (Fig. 4). Subsequently, the patient became hemodynamically stable and a vena cava filter insertion was followed under fluoroscopy in his inferior vena cava via internal jugular vein approach. It was decided to treat the acetabulum fracture conservatively and the patient was admitted to the intensive care unit immediately after surgery with systemic heparinization. His general condition was completely restored after a week stay in to the intensive care unit and he was transferred to a ward. After 1 month, he was discharged from the hospital. After 3 months, he could walk by himself although a slight limp remained. On his latest follow up after 2 years, the radiography showed the acetabulum fractures were united completely, but also showed osteoarthritis changes on his right hip joint.

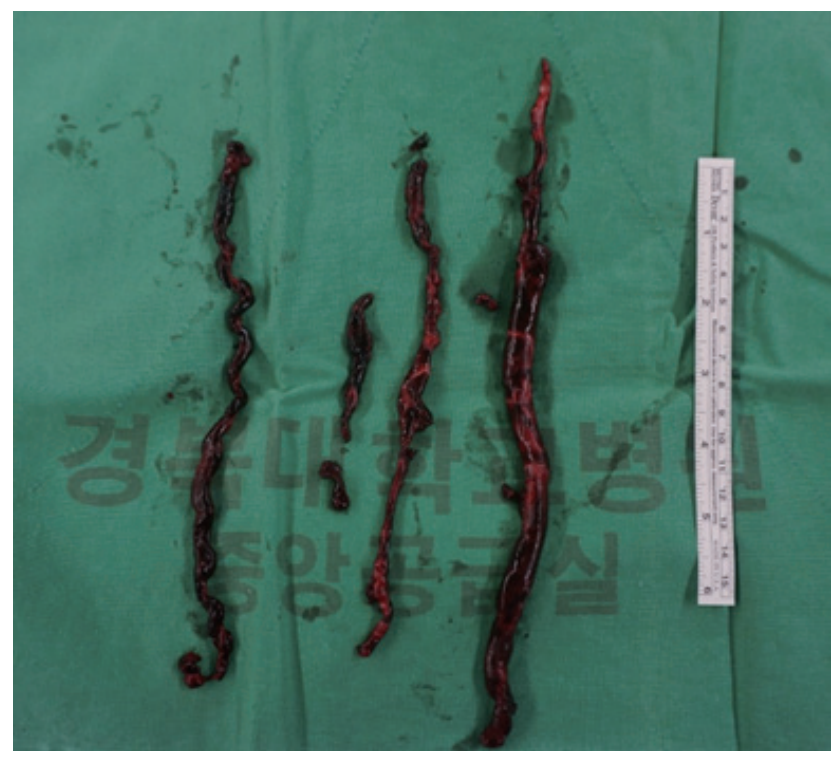

Fig. 4. Four pieces of large emboli were evacuated.

\section{DISCUSSION}

Known predisposing risk factors of the VTE are linked to age greater than 60 , malignancy, pregnancy, previous history of DVT or PTE, oral contraceptives, prolonged operation time, spinal cord injury, and obesity $[1,5,6]$. Patients with pelvic or acetabular trauma are known to be at increased risk for the development of thromboembolic complications. In patients with pelvic and acetabular fractures, the risk of pulmonary embolism is $2 \%$ to $10 \%$ with subsequent death occurring in $0.5 \%$ to $2 \%$ of patients [7]. Moreover, thromboembolic complications may occur more commonly in patients with a major pelvic disruption, especially if the associated extremity fractures [7].

In the current case, the patient had several risk factors including advanced age, simultaneous pelvic fracture, and 10 days of immobilization, although he had no subjective symptoms or objective signs of DVT.

Most pulmonary emboli does not shows any symptoms and the pulmonary complication of trauma such as lung contusion may obscure the presentation of pulmonary embolism resulting in a delay in diagnosis or misdiagnosis [3].

In this case, the patient had no pulmonary symptoms but the cause of embolus can be attributed to the long duration of immobilization because the evacuated emboli were the shape of deep veins in lower extremity. It is assumed that the cause of PTE in this case was compression of lower extremity with an elastic bandage while preparing for the operation and manipulating the lower extremity. It is thought that thrombi formed within the deep vein of the leg while mobilized and floated to pulmonary artery via the right heart.

Because of high risk of thromboembolic phenomena in multiple trauma patients and effective medical prophylaxis and protection are not established, many authors advised the administration of a vena cava filter $[4,6,8]$. Modern vena cava filters are known to be relatively safe and easy to insert acting as an effective mechanical barrier to filter the thrombi off, especially in trauma patients [8]. Although the vena cava filter was placed after embolectomy to prevent possible remained thrombi from floating in current case, it is thought that this procedure should have been done in advance of the surgical intervention. It 
has been well established that elderly patients with pelvic ring or acetabular fractures are at greater risk of thrombosis. The majority of reports on the subject have concluded that age significantly affects the development of VTE [1,7]. Accordingly, age appears to be an important cause of VTE. Thus, it is necessary to consider chemoprophylaxis as well as mechanical prophylaxis, such as anticoagulant agents, in elderly patients with high energy injuries and acetabular injury. Fortunately, the patient's life could be saved because the PTE was not detected during surgery but strong suspicious PTE, so CT scan was done under general anesthesia, and an immediate embolectomy could be done after scanning.

Nobody knows what would happen if the symptom emerged in the ward or elsewhere out of the operating room. As far as we know, there has been few report that a patient's life had been saved after PTE happened during acetabular operation. Preoperative screening, such as DVT-CT or duplex ultrasonography, will be useful, especially in patients with the risk factors. If DVT is detected, preoperative insertion of vena cava filter or administration of thrombolytic agent will be a great help to prevent PTE. Some method of prophylaxis should be considered based on a patient's overall clinical situation.

In conclusion, PTE is a fatal complication. Preoperative screening and mechanical and chemical prophylactic management is highly advised, especially in high risk factor patients. We cannot stress enough to be cautious and keep patients informed of possible complications that occur. When even during an operation a suspicious unstable sign occurs, do not hesitate to conduct further evaluation of thromboembolism such as CT scan.

\section{REFERENCES}

1. Geerts WH, Bergqvist D, Pineo GF, Heit JA, Samama CM, Lassen MR, et al. Prevention of venous thromboembolism: American College of Chest Physicians evidence-based clinical practice guidelines (8th Edition). Chest 2008;133(6 Suppl):381S-453S.

2. Hill J, Treasure T; Guideline Development Group. Reducing the risk of venous thromboembolism (deep vein thrombosis and pulmonary embolism) in patients admitted to hospital: summary of the NICE guideline. Heart 2010;96:879-82.

3. O'Malley KF, Ross SE. Pulmonary embolism in major trauma patients. J Trauma 1990;30:748-50.

4. Webb LX, Rush PT, Fuller SB, Meredith JW. Greenfield filter prophylaxis of pulmonary embolism in patients undergoing surgery for acetabular fracture. J Orthop Trauma 1992;6:139-45.

5. Coon WW. Risk factors in pulmonary embolism. Surg Gynecol Obstet 1976;143:385-90.

6. Dennis JW, Menawat S, Von Thron J, Fallon WF Jr, Vinsant GO, Laneve LM, et al. Efficacy of deep venous thrombosis prophylaxis in trauma patients and identification of high-risk groups. J Trauma 1993;35:132-8; discussion 138-9.

7. Montgomery KD, Geerts WH, Potter HG, Helfet DL. Thromboembolic complications in patients with pelvic trauma. Clin Orthop Relat Res 1996;(329):68-87.

8. Smoot RL, Koch CA, Heller SF, Sabater EA, Cullinane DC, Bannon MP, et al. Inferior vena cava filters in trauma patients: efficacy, morbidity, and retrievability. J Trauma 2010;68:899-903. 